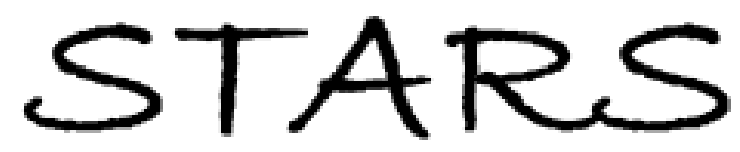

University of Central Florida

STARS

$1-1-1995$

\title{
Micro-Raman Spectroscopy In The Undergraduate Research Laboratory
}

R. Voor

University of Central Florida

L. Chow

University of Central Florida

A. Schulte

University of Central Florida

Find similar works at: https://stars.library.ucf.edu/facultybib1990

University of Central Florida Libraries http://library.ucf.edu

This Article is brought to you for free and open access by the Faculty Bibliography at STARS. It has been accepted for inclusion in Faculty Bibliography 1990s by an authorized administrator of STARS. For more information, please contact STARS@ucf.edu.

\section{Recommended Citation}

Voor, R.; Chow, L.; and Schulte, A., "Micro-Raman Spectroscopy In The Undergraduate Research Laboratory" (1995). Faculty Bibliography 1990s. 1230.

https://stars.library.ucf.edu/facultybib1990/1230

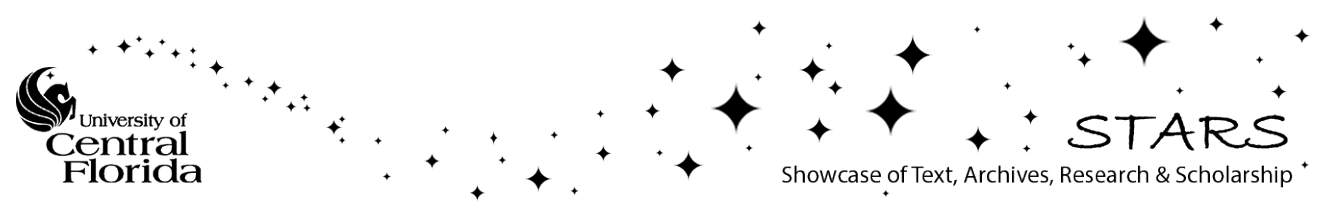




\section{Micro-Raman spectroscopy in the undergraduate research laboratory}

R. Voor, L. Chow, and A. Schulte

Citation: American Journal of Physics 62, 429 (1994); doi: 10.1119/1.17544

View online: https://doi.org/10.1119/1.17544

View Table of Contents: https://aapt.scitation.org/toc/ajp/62/5

Published by the American Association of Physics Teachers

\section{ARTICLES YOU MAY BE INTERESTED IN}

High performance Raman spectroscopy with simple optical components

American Journal of Physics 78, 671 (2010); https://doi.org/10.1119/1.3427413

A nondestructive tool for nanomaterials: Raman and photoluminescence spectroscopy

American Journal of Physics 73, 224 (2005); https://doi.org/10.1119/1.1819933

Raman scattering spectroscopy of liquid nitrogen molecules: An advanced undergraduate physics laboratory experiment

American Journal of Physics 75, 488 (2007); https://doi.org/10.1119/1.2721584

A simple apparatus for observing the Raman effect

American Journal of Physics 58, 893 (1990); https://doi.org/10.1119/1.16501

Rotational Raman scattering in the instructional laboratory

American Journal of Physics 62, 639 (1994); https://doi.org/10.1119/1.17484

Zeeman effect experiment with high-resolution spectroscopy for advanced physics laboratory

American Journal of Physics 85, 565 (2017); https://doi.org/10.1119/1.4984809

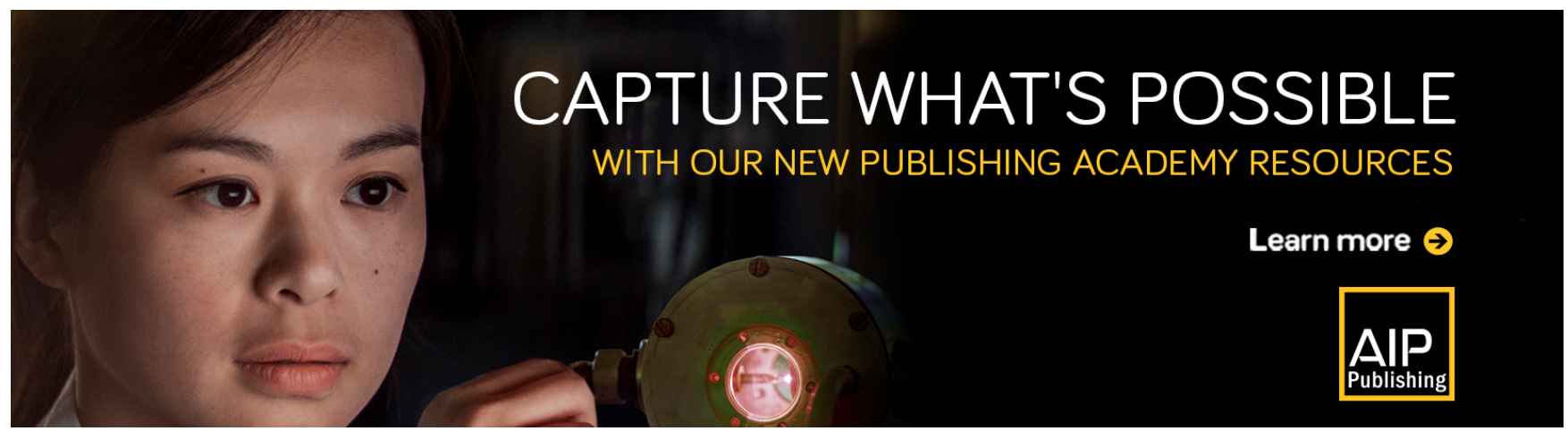




\section{CONCLUSION}

It is now quite clear that the ellipsoid of revolution in Fig. 1 has the same relationship with the $\bar{S}$ wave front as that of Fig. 2 with the $S$ wave front. Indeed if one substitutes the inversions of Eqs. (17) into Eqs. (7)-(9), one obtains the form of Eqs. (12)-(15) with $\beta \rightarrow-\beta$. The ellipsoid of Fig. 1 is the spatial locus of object wave front events in $\bar{S}$, and the ellipsoid of Fig. 2 is the spatial locus of image wave front events in $S$. They are, respectively, made up of minor circles of $\bar{S}$ wave front events and $S$ wave front events as these two spherical wave fronts expand radially at the speed of light from their respective origins. Thus, while the relativity principle is not violated, the relationship between the $S$ and $\bar{S}$ wave fronts is a bit more subtle than just a Lorentz transformation. It is only by the time delays and advances on rays from the object wave front that one obtains a set of image events in $S$ that are symmetrically distributed about the origin of $\bar{S}$. Then, upon relabeling by a Lorentz transformation, the image events become simultaneous wave front events in $\bar{S}$ and the ellipsoid is contracted into a sphere centered on the origin $\bar{O}$.

\footnotetext{
a) email: w.moreau @ csc.canterbury.ac.nz

${ }^{1}$ A. Einstein, "Zur elektrodynamik bewegter Körper," Ann. Phys. 17, 891-921 (1905); translation in The Relativity Principle (Dover, New York), p. 46.

${ }^{2}$ A. P. French, Special Relativity (Norton, New York, 1968), pp. 81-82. ${ }^{3} \mathrm{C}$. Moller, The Theory of Relativity (Oxford, London, 1972), pp. 37-39. ${ }^{4}$ W. G. V. Rosser, An Introduction to the Theory of Relativity (Butterworths, London, 1964), pp. 89-90; in a later section of his book, pp. 122-125, Rosser gives a discussion of the topic presently under consideration, but his analysis is limited to a single light ray in the direction of the motion of $\bar{S}$ with respect to $S$.

${ }^{5}$ W. Rindler, Special Relativity (Interscience, New York, 1960), pp. 16-19.

${ }^{6}$ W. Pauli, Theory of Relativity (Pergamon, London, 1958), pp. 9 and 10. ${ }^{7}$ C. W. Misner, K. S. Thorne, and J. A. Wheeler, Gravitation (Freeman, San Francisco, 1973), pp. 715-717.
}

\title{
Micro-Raman spectroscopy in the undergraduate research laboratory
}

\author{
R. Voor, L. Chow, and A. Schulte ${ }^{\text {a) }}$ \\ Department of Physics, University of Central Florida, Orlando, Florida 32816-2385
}

(Received 14 June 1993; accepted 19 November 1993)

\begin{abstract}
Modern materials science requires processing and characterization techniques for microscopic structures. Molecular probes such as Raman spectroscopy are some of the most viable tools, particularly if they are supplemented by imaging to obtain spatially resolved compositional information of inhomogeneous or low volume samples. In order to introduce these techniques and materials science experiments into the advanced undergraduate laboratory, we have constructed an inexpensive micro-Raman attachment, which consists of an off-the-shelf microscope and the coupling optics to an existing Raman spectrometer. The modification of the microscope, the optical coupling, and a low cost viewing system for positioning the laser excitation on the sample are described in detail. The students study molecular spectra of new materials such as diamond films, Fullerenes, and biological compounds with spatial resolution of several microns.
\end{abstract}

\section{INTRODUCTION}

Raman scattering - or scattering of light at altered frequency as first described by Raman and Krishnan ${ }^{1}$-yields structural and dynamic information on a molecular level. ${ }^{2,3}$ As a probe it is nondestructive and therefore it is one of the most important tools for characterization of new materials. Due to recent simplification in the design of modern Raman detection systems, ${ }^{4}$ they are becoming an option for the budget of an advanced undergraduate research laboratory. However, a search through the volumes of this journal during the last decade shows very few publications concerned with the application of modern spectroscopic techniques, and only a note which deals with Raman scattering. ${ }^{5}$ In this paper we present Raman spectroscopic experiments to introduce students to light scattering techniques and to state of the art applications in materials science. We describe a setup, which has the advantage that the laser spot on the sample can be imaged in situ and that microscopic regions of a material under study can be easily probed.

In a typical Raman experiment, the excitation source is a laser, and the scattered light is analyzed by a spectrometer and a detector with sensitivity near the single photon level. The inelastically scattered light contains information on vibrational states of the sample, which manifests itself by a frequency shift from the incident light. The underlying physics is that vibrations (or other excitations) modulate the polarizability tensor and cause the induced dipole moment to radiate at frequencies different from the electric field vector of the incoming light wave. For most applications the spontaneous Raman scattering originating from the linear response to the electric field is measured. The 
experimental challenge is to detect the weak Raman signal while rejecting the intense Rayleigh background. Recently, the development of efficient filters for Rayleigh rejection and the availability of multichannel detection have considerably simplified the experimental setup with the additional benefits of increased optical throughput and shorter acquisition time. The spectrometer can be reduced to a single spectrograph stage with a notch or sharp cut-off filter selected for high extinction at the Rayleigh line. ${ }^{4}$ Thus Raman spectroscopy has become even more accessible as a scientific tool. From an analytical point of view, it is often desirable to analyze samples in nano- or picogram quantities. These requirements can be met by combining an optical microscope with a Raman system. Then the excitation spot has a dimension in the micron range, and $\mathrm{Ra}$ man spectroscopy provides molecular compositional information with high spatial resolution.

A key element (and one of the most expensive parts) in micro-Raman spectrometers is the microscope attachment, which includes a viewing system to position the laser beam on the sample. Motivated by the high potential and the large information content of Raman microspectroscopy, we have carried out an undergraduate project to convert an existing surface finish microscope to a Raman microprobe. In this article we describe the construction of the microscope attachment using only standard and easily available parts. We then present experiments to characterize small quantities of protein powders and Fullerenes. In addition, the microstructure of diamond films is investigated.

\section{ELEMENTARY THEORETICAL BACKGROUND ON RAMAN SCATTERING}

A classical treatment of Raman scattering can be obtained for a simple diatomic molecule by approximating the binding effect of the electronic charge distribution by a spring between its nuclei. ${ }^{2}$ For linear molecules the number of possible vibrations is $(3 N-5)$ [for nonlinear molecules $(3 N-6)]$ where $N$ is the number of atoms. Accordingly, the nuclei are space and rotation fixed about their equilibrium position but free to vibrate in simple harmonic motion along one normal coordinate $Q$ as follows:

$$
Q=Q_{0} \cos (\omega t),
$$

where $\omega$ is the angular frequency of vibration of the molecule. The polarizability of the molecule is dependent on the position of the nuclei, and this can be expressed by expanding the dielectric polarizability with respect to $Q$ in a Taylor series as

$$
\boldsymbol{\alpha}=\boldsymbol{\alpha}_{0}+Q\left(\frac{\partial \boldsymbol{\alpha}}{\partial Q}\right)_{0}+\frac{Q^{2}}{2}\left(\frac{\partial^{2} \alpha}{\partial Q^{2}}\right)_{0}+\frac{Q^{3}}{6}\left(\frac{\partial^{3} \alpha}{\partial Q^{3}}\right)_{0}+\cdots,
$$

where $\alpha$ is the polarizability tensor of the molecule and the subscript " 0 " on the derivatives indicates that they are to be evaluated at the equilibrium configuration. Linearly approximating the effect of the moving nuclei with the first two terms of the expansion and substituting the expression for $Q$ yields

$$
\alpha=\alpha_{0}+Q_{0} \cos (\omega t)\left(\frac{\partial \alpha}{\partial Q}\right)_{0}
$$

When an electromagnetic wave is incident on the molecule, it induces a dipole moment $\mathbf{P}$ in the electron distribution, which in the linear approximation is given by

$$
\mathbf{P}=\boldsymbol{\alpha} \mathbf{E} \text {. }
$$

$\mathbf{E}$ is the $\mathbf{E M}$ wave given by

$$
\mathbf{E}=\mathbf{E}_{0} \cos \left(\omega_{1} t\right) \text {. }
$$

The induced dipole moment then becomes

$$
\mathbf{P}=\boldsymbol{\alpha}_{0} \mathbf{E}_{0} \cos \left(\omega_{1} t\right)+\left(\frac{\partial \alpha}{\partial Q}\right)_{0} \mathbf{E}_{0} Q_{0} \cos \left(\omega_{1} t\right) \cos (\omega t) .
$$

This can be written as

$$
\begin{aligned}
\mathbf{P}= & \alpha_{0} \mathbf{E}_{0} \cos \left(\omega_{1} t\right)+\left(\frac{\partial \alpha}{\partial Q}\right)_{0} \mathbf{E}_{0} Q_{0} \frac{1}{2}\left\{\cos \left[\left(\omega_{1}+\omega\right) t\right]\right. \\
& \left.+\cos \left[\left(\omega_{1}-\omega\right) t\right]\right\} .
\end{aligned}
$$

The induced dipole moment is a function of the frequencies of the incident electromagnetic radiation and the vibration of the molecule causing the Rayleigh scattered light of frequency $\omega_{1}$ and the Stokes and anti-Stokes Raman scattering of frequencies $\left(\omega_{1}-\omega\right)$ and $\left(\omega_{1}+\omega\right)$, respectively. Equation (7) also shows that the selection rule for Raman activity of a vibrational mode is a nonvanishing derivative of the polarizability with respect to the normal coordinate of that mode.

The above equations can be generalized to the case where the molecular polarizabilities are not isotropic and the induced dipole moment vector points in a different direction than the electric field vector. ${ }^{2}$ A more profound quantum mechanical treatment can relate the polarizability tensor to the wave functions and the scattering levels of the scattering system. ${ }^{2}$ Similar to Rayleigh scattering, in the absence of nonlinear effects the intensity of the scattered Raman light increases with the fourth power of the frequency of the exciting radiation.

\section{EXPERIMENTAL APPARATUS}

The design of the micro-Raman system developed in this project is essentially a modification of the fore optics of a modern Raman spectrometer. The main components are: the microscope including viewing system, the laser, spectrograph, and detector. A schematic of the setup is shown in Fig. 1. The Raman system employs an argon ion laser as the excitation source and a single grating spectrograph (Instruments SA model HR320) equipped with a 1200 line $/ \mathrm{mm}$ grating, which gives a dispersion of $2.4 \mathrm{~nm} / \mathrm{mm}$ at the focal plane. Rayleigh line rejection is achieved using a holographic notch filter (Kaiser Optical Systems model HNF-514-1.0).

The scattered light is detected with a charge coupled device (CCD) system. The CCD itself is a Si-based array detector. Absorbed photons are converted to electron-hole pairs. The electrons are collected in potential wells created by a depletion layer. The wells can be addressed individually and digitized with the readout electronics. CCDs are characterized by a high dynamic range $\left(\approx 10^{5}\right)$, high quantum efficiency $(\approx 50 \%)$, wide spectral range $(400$ $1050 \mathrm{~nm}$ ), and low readout noise ( $\left.\approx 10 e^{-} / \mathrm{pixel}\right)$. CCDs have become the detector of choice for most Raman systems, and their prices have recently become competitive with those of lower performance detectors usually found in undergraduate research labs. 


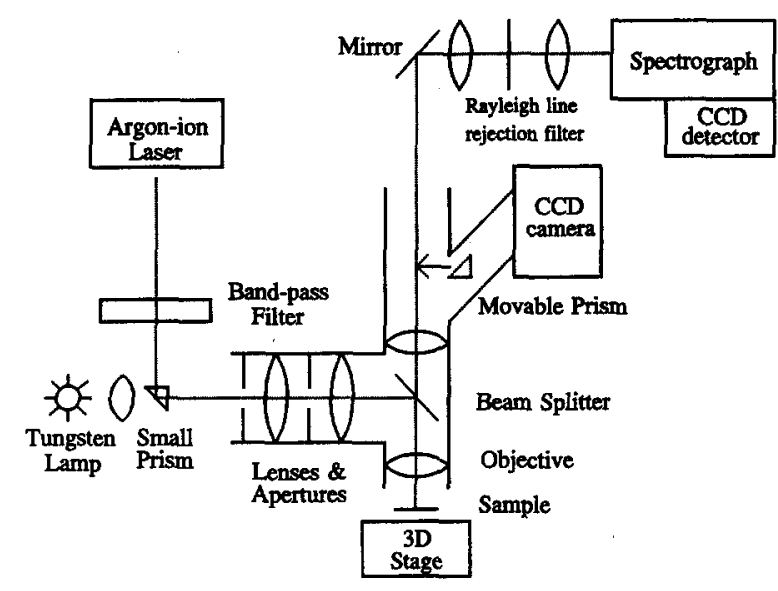

Fig. 1. Schematic diagram of the apparatus for micro-Raman spectroscopy.

The micro-Raman attachment was constructed as part of an undergraduate research project. ${ }^{6}$ The design goals were to bring the laser beam into the microscope, focus the beam to a spot with diameter of a few microns, collect the light scattered near $180^{\circ}$ using the microscope's objective lens, and then deliver the scattered light to the spectrometer. The imaging of the sample and the excitation spot must be maintained throughout.

We had a Surface Finish Microscope (Nikon model 70048) with an image-plane design and a tube length of $170 \mathrm{~mm}$ available. The "off-the-shelf" unit provided the basic structure, namely the vertical tube, beamsplitter, objectives, switchable exit ports, and a tungsten lamp illumination. To this we added the coupling optics for the laser beam, a stable sample positioning stage, and an inexpensive viewing system. Since the microscope was originally designed for surface finish applications, it has no substage condenser for sample illumination. The illumination comes from a tungsten lamp focused through the objective lens. Since the microscope has an image-plane design and is not infinity corrected, focusing is done by moving the sample stage vertically, keeping the distance from the objective to the spectrograph slit fixed. In order to couple the laser beam into the microscope and at the same time maintain good imaging, we use a right-angle prism $\left(5 \times 5 \times 3 \mathrm{~mm}^{3}\right.$ base, Melles Griot) mounted in front of the tungsten lamp such that both the laser beam and the broadband illumination will follow the same optical path through the microscope. The laser beam and thus the microscope housing are at a fixed height. Therefore focusing is done by moving the sample. An adaptor was machined from aluminum to mount a combination of one vertical and two horizontal micrometer stages to the microscope base. This construction provides the necessary mechanical stability to minimize vibrations and $x-y-z$ micropositioning of the sample in the laser excitation.

Inside the microscope, the incoming beam is deflected by a standard beamsplitter made from a thin dielectric coating on a fused silica substrate. We employed the original $45^{\circ}$ beamsplitter, which was designed for the visible range of the spectrum and proved to be quite adequate to handle the incoming laser radiation of typically $100 \mathrm{~mW}$. The beamsplitter has a reflection/transmission ratio of about $1 / 10$ at the argon-ion laser wavelength of $514 \mathrm{~nm}$, and is sensitive to the polarization of the incident light. The argon-ion laser beam is linearly polarized, and a half-wave plate can be used to rotate the polarization plane for the beamsplitter's optimum reffection coefficient. This maximizes the throughput of the incident light, whereas no polarization analysis of the scattered light is performed. The polarization of the laser was checked by monitoring the symmetric stretching vibration $\left(459 \mathrm{~cm}^{-1}\right)$ of carbon tetrachloride.

The microscope has two exit ports, one of which is used for the camera attachment, the other for directing the Raman signal to the spectrograph. The switch between the two is controlled by a prism moving in and out of the optical path. When combining a laser and a microscope some safety precautions are mandatory. Safety goggles should be worn at all times when aligning the laser beam and the coupling optics. In particular, one must be aware that laser radiation can be reflected through the exit ports. Therefore the port deflecting the Raman scattered light is covered by a $45^{\circ}$ mirror. The reflected laser light must be prevented from accidentally hitting the observer's eye through the ocular, and therefore the eyepiece is replaced by a CCD camera which is mounted with a relay adaptor (Edmund Scientific). The relatively inexpensive imaging system (CCD camera: Electrim model EDC-1000, $\approx \$ 800$ ) is interfaced through a half-size expansion card to an IBM-compatible computer. The active area of the CCD chip contains $754 \times 488$ pixels with an 8 bit digital output. Images are stored in Tag Image File Format (TIFF) files and are readily processed with accompanying software. This type of CCD camera has also found applications in astronomy, photometry, and industrial inspection.

The scattered light from the sample is collected by the objective (Oriel, power $20 \times$, NA 0.4 ) and transmitted through the beamsplitter to the second exit port, when the prism is in the "out" position. A singlet of $260 \mathrm{~mm}$ focal length collimates the Raman scattered light. The collimated beam is then focused by a $f / 5$ biconvex lens onto the slit matching the $f$ number of the spectrograph.

Each spectrum shown in Figs. 2-4 was taken with 514 $\mathrm{nm}$ excitation from an argon-ion laser at a spectral resolution of $5 \mathrm{~cm}^{-1}$ ( 1200 grooves $/ \mathrm{mm}$ grating). The wavenumber shift of the Stokes Raman scattered light is plotted relative to the laser line. For certain objectives weak band from the antireflection coatings are seen at 846, 1255, and $1297 \mathrm{~cm}^{-13}$. Emissions from the computer monitor and room light are eliminated by working in the dark.

\section{MICRO-RAMAN CHARACTERIZATION OF SMALL PARTICLES}

Analytical applications in biotechnology and chemistry often require the characterization of samples, which are only available in minuscule quantities. Using the microRaman attachment we can obtain vibrational spectra from micron-size particles. We have chosen the protein lysozyme to demonstrate this feature, since it is readily available, highly stable, and a classic example for application of Raman spectroscopy to biopolymers. This protein is a small enzyme with a single polypeptide chain of 129 residues cross linked by four disulfide bridges. Lyophilized hen egg white lysozyme was obtained from Sigma Chemical Co. (crystallized three times) and used without further purification. Figure 2(a) shows the image of the lysozyme particle exposed in this analysis. The dimension is indi- 


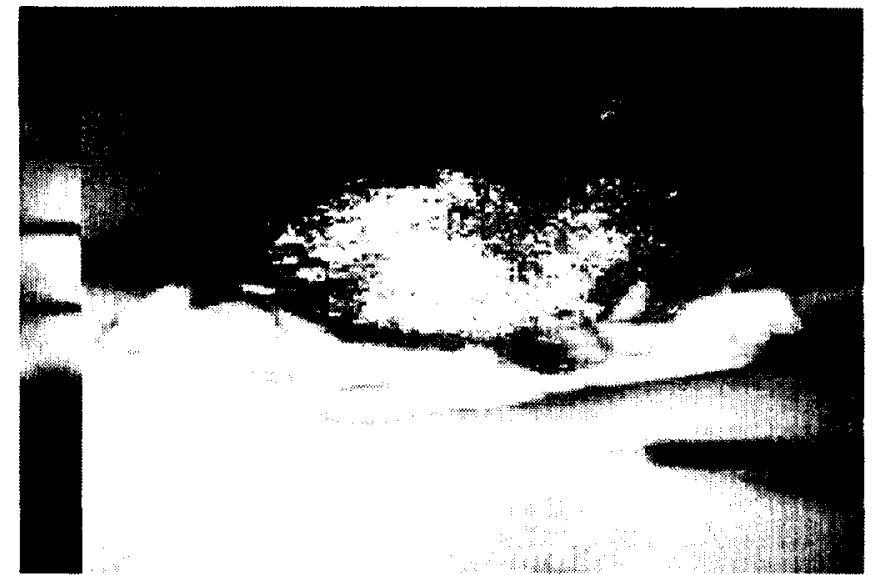

(a)

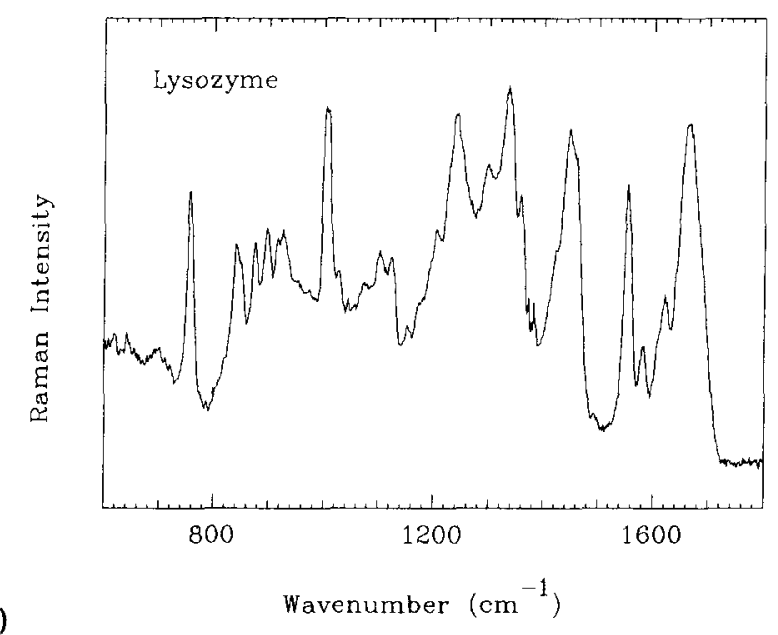

Fig. 2. (a) Image of a lysozyme grain. One division of the scale bar is equal to $25.4 \mu \mathrm{m}$. (b) Raman spectrum of the lysozyme grain shown in (a).

cated by a scale bar with each division equal to $25.4 \mu \mathrm{m}$. The Raman spectrum taken with a $100 \mathrm{~s}$ exposure time and relatively low laser power of $18 \mathrm{~mW}$ at the sample is shown in Fig. 2(b). The band positions are characteristic for the native protein, and the assignments can be found in the literature. ${ }^{7}$ There are sharp lines due to constituent amino acids with aromatic sidegroups, such as indole-ring vibrations of tryptophan near 760 and $1012 \mathrm{~cm}^{-1}$. The bands near $1260 \mathrm{~cm}^{-1}$ (amide III) and $1660 \mathrm{~cm}^{-1}$ (amide I) correspond to frequencies of the peptide CONH groups. These vibrations are sensitive to protein conformation and are indicative for the native state.

Biological materials may find a place in the molecular electronics of the future. For instance, it has been demonstrated that the photosensitive membrane protein bacteriorhodopsin can make an outstanding switching device. ${ }^{8}$ Such assemblies are often manufactured as thin films. Extensions of our experiments to these areas can be envisioned. Micro-Raman is a valuable probe, since it yields spatial information across the device and can identify impurities and defects. Investigation of components and molecules in living cells is another emerging application of Raman microspectroscopy.

The capability of our micro-Raman system to characterize $\mu \mathrm{g}$ quantities of newly synthesized compounds is demonstrated for clustered forms of carbons such as $\mathrm{C}_{60}$ and $\mathrm{C}_{70}$. These compounds belong to a new class of geodesic

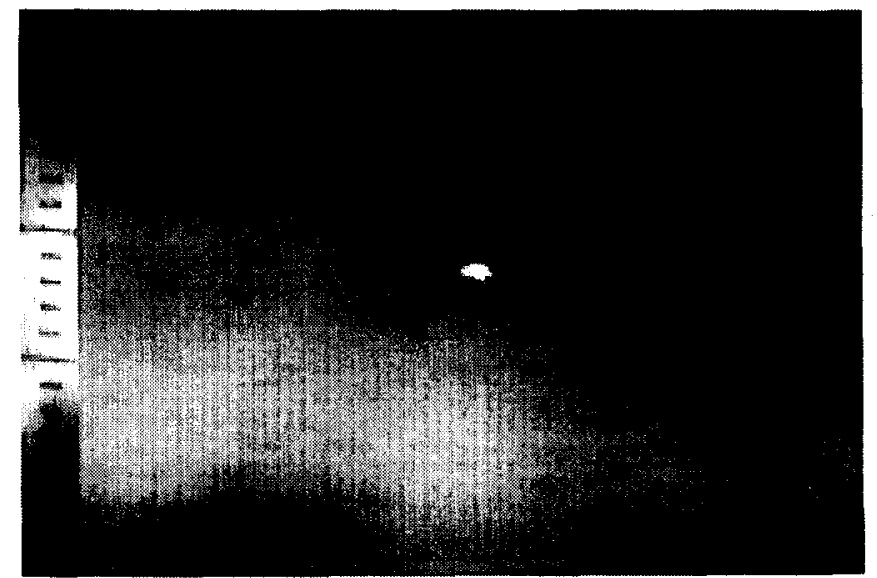

(a)

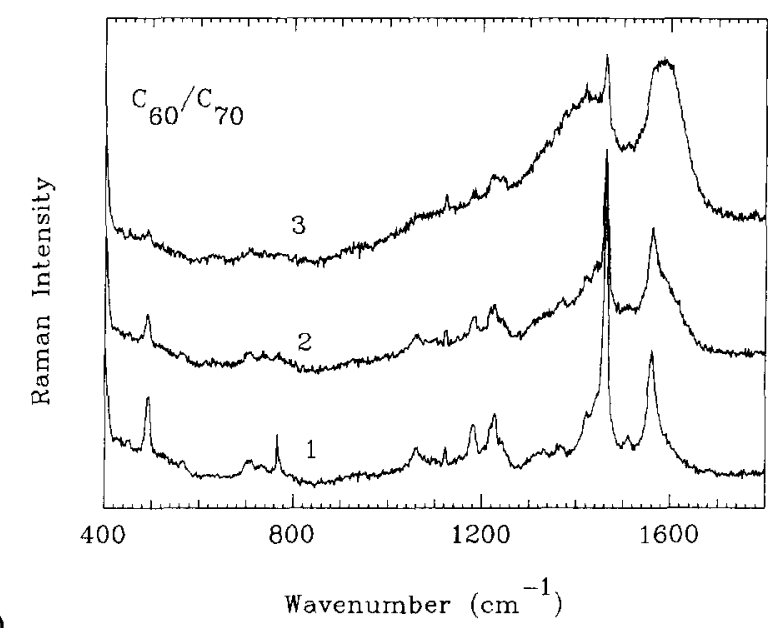

Fig. 3. (a) Image of $\mathrm{C}_{60} / \mathrm{C}_{70}$ powder particles. One small division of the scale bar corresponds to $25.4 \mu \mathrm{m}$. (b) Raman spectrum of $\mathrm{C}_{60} / \mathrm{C}_{70}$ powder. Spectra (1)-(3) were taken at the same spot $130 \mathrm{~s}$ apart using $3 \mathrm{~mW}$ power at the sample and $100 \mathrm{~s}$ exposure time. The broadening of the spectral bands with increasing laser irradiation indicates structural changes in the sample.

cage molecules termed Fullerenes. Initially produced in 1985 from laser vaporization of graphite, research in this area has recently been intensified by the availability of carbon Fullerenes in macroscopic quantities. ${ }^{9}$ Micro-Raman scattering is particularly suited to the analysis of carbon Fullerenes since it provides characterization of low volume samples. A commercially available mixture of $\mathrm{C}_{60}$ and $\mathrm{C}_{70}$ powder (Texas Fullerenes Corp.) was analyzed. Raman spectroscopy was performed on as-received samples to determine the degree of purity. Figure 3 (a) shows the excitation spot on the sample. Spectra [Fig. 3(b)] were taken at the same spot about $130 \mathrm{~s}$ apart with a $100 \mathrm{~s}$ exposure time and $3 \mathrm{~mW}$ of power at the sample. The Raman spectrum is a probe of the normal mode frequencies of the $\mathrm{C}_{60}$ and $C_{70}$ cages, and the vibrational bands have been assigned based on the molecular symmetries. The band at $1463 \mathrm{~cm}^{-1}$ arises from both $C_{60}$ and $C_{70}$, the $1561 \mathrm{~cm}^{-1}$ peak indicates $C_{70}$, and the $491 \mathrm{~cm}^{-1}$ line is due to $C_{60} \cdot{ }^{10}$ Extended irradiation with the $514 \mathrm{~nm}$ argon line [spectra 2 and 3 in Fig. 3(b)] causes widening or disappearance of these bands indicating a photo- or thermally induced transition ${ }^{11}$ due to the focused laser beam. 


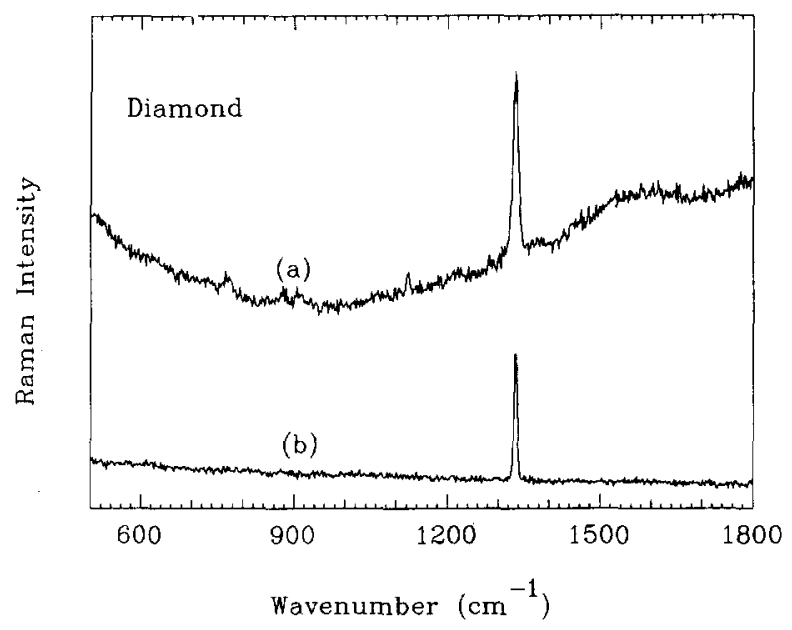

Fig. 4. Micro-Raman spectra of a CVD deposited diamond thin film (a) and of crystalline diamond powder (b).

\section{STRUCTURAL STUDIES OF DIAMOND THIN FILMS}

In recent years, growth of diamond thin films by chemical vapor deposition (CVD method) has been a very active research field. ${ }^{12}$ All these activities are fueled by the promise of potential applications of diamond thin films, which include heat sinks for high power microelectronic devices and coatings for abrasive tools and for high temperature, and high damage threshold electronic devices.

Micro-Raman spectroscopy has been proven to be the most suitable technique for characterizing diamond thin films ${ }^{13}$ because of its ability to distinguish crystalline diamond, amorphous diamond, and other graphitic soot or charcoal by virtue of their different vibrational modes in different structures. The Raman scattering efficiency for the $s p^{2}$-bonded graphite is more than 50 times greater than that for the $s p^{3}$-bonded diamond, therefore, small amounts of $s p^{2}$-bonded carbon in the diamond deposits can be readily detected.

Our diamond thin films were grown using hot-filament CVD method. ${ }^{14}$ In Fig. 4, the spectrum (a) is of a diamond thin film grown in our laboratory using hot-filament CVD. (b) is the spectrum of a $40 \mu \mathrm{m}$ natural diamond particle. The spectrum of the natural diamond is dominated by a strong and narrow peak at $1333 \mathrm{~cm}^{-1}$. This peak corresponds to the carbon-carbon stretching due to the motion of the two fcc sublattices, which form the diamond crystal structure. Indeed the CVD diamond thin film shows a sharp band at $1333 \mathrm{~cm}^{-1}$, which is indicative for the diamond structure. The spectrum displays only a small degree of graphite contamination ( $s p^{2}$ bonding) corresponding to the weak background around $1580 \mathrm{~cm}^{-1}$.

\section{CONCLUSIONS}

We have described a home-built micro-Raman attachment suitable for advanced undergraduate research projects. The performance is illustrated by studies of materials of technological interest such as diamond films and carbon Fullerenes as well as small amounts of protein. Micro-Raman spectra provide molecular compositional information of samples with a size in the micron range. $\mathrm{Em}$ - ploying a microscope also allows us to image the specimen in situ and to position the laser excitation beam.

Raman and infrared absorption spectroscopy are complementary ways to measure molecular vibrations, and are subject to different selection rules. For Raman spectroscopy there must be a change of polarizability during the vibration as opposed to a change in dipole moment for an IR active vibration. Raman spectroscopy is important for biological samples, since it is not hindered by water absorption. The whole vibrational spectrum from several to a few thousand wave numbers can be obtained without changing beamsplitters and detectors. Absorption spectroscopy often has a higher detection limit (though Raman scattering can be resonance enhanced), and usually fluorescence emission is not a problem. Raman spectroscopy is easily adaptable to microsamples, since both the probe light and the viewing system use the same spectral range. The examples demonstrate that in most cases no special samples preparation is required. The polarization dependence of Raman spectra relates to the molecular symmetry even in liquids, which is useful for band assignments.

There is a wide range of problems which can be studied with the described microspectroscopy apparatus. For instance, the same setup can be employed to study fluorescence phenomena. In our department, there is considerable interest in solid state laser materials. The fluorescence spectrum contains information on possible lasing transitions and site symmetry of the dopants. Since the fluorescence in these materials is quite strong, a less sensitive detector such as an unintensified diode array could be employed.

\section{ACKNOWLEDGMENTS}

Partial support of the project comes from Grant No. 11-68-920 from Division of Sponsored Research, University of Central Florida. One of the authors (R.V.) would like to acknowledge support by the National Science Foundation's Research Experience for Undergraduates program through the Center for Research and Education in Optics and Lasers.

\footnotetext{
a) Author to whom correspondence should be addressed.

'C. V. Raman and K. S. Krishnan, "A new type of secondary radiation," Nature 121, 501 (1928).

${ }^{2}$ D. A. Long, Raman Spectroscopy (McGraw-Hill, New York, 1977).

${ }^{3}$ D. J. Gardiner and P. R. Graves, Practical Raman Spectrascopy (Springer, Berlin, 1989).

${ }^{4}$ T. M. Niemczyk, M. Delgado-Lopez, and D. C. Newman, "Raman spectroscopy tackles industrial problems," Laser Focus 29, 85-98 (1993).

${ }^{5}$ R. Feinberg, "A simple apparatus for observing the Raman effect," Am. J. Phys. 58, 893 (1990).

${ }^{6} \mathbf{R}$. Voor, "Construction and Application of a Micro-Raman Spectrometer," University of Central Florida, Honor's thesis, 1992 (unpublished).

${ }^{7}$ R. C. Lord and N.-T. Yu, "Laser-excited Raman spectroscopy of biomolecules," J. Mol. Biol. 50, 509-524 (1970).

${ }^{8}$ G. A. Schick, A. F. Lawrence, and R. R. Birge, "Biotechnology and molecular computing," Trends Biotechnol. 6, 159-163 (1988).

${ }^{9}$ W. Krätschmer, L. D. Lamb, K. Fostiropoulos, and D. R. Huffman, "Solid C60: A new form of carbon," Nature 347, 354-358 (1990).

${ }^{10}$ D. S. Bethune, G. Meijer, W. C. Tang, and H. J. Rosen, "The vibrational Raman spectra of purified solid films of $\mathrm{C}_{60}$ and $\mathrm{C}_{70}$ " Chem. Phys. Lett. 179 219-222 (1990).

${ }^{11}$ J. D. Robertson, E. Eloi, M. S. Meier, S. L. Ren, X.-X. Bi, P. C. Eklund, and M. S. Dresselhaus, "Photo-assisted structural transition and oxygen
} 
diffusion in solid $\mathrm{C}_{60}$ films," Appl. Phys. Lett. 60, 2871-2873 (1992).

${ }^{12} \mathrm{~K}$. Spear, "Diamond-ceramic coating of the future," J. Am. Ceram. Soc. 72, 171-191 (1989).

${ }^{13}$ D. S. Knight and W. B. White, "Characterization of diamond films by
Raman spectroscopy," J. Mater. Res. 4, 385-393 (1989).

${ }^{14}$ L. Chow, A. Horner, H. Sakouri, B. Roughani, and S. Sundaram, "Growth of $(100)$ oriented diamond thin films on ball structure diamond-like particles," J. Mater. Res. 7, 1606-1609 (1992).

\title{
Scaling properties in the diffraction of focused waves and an application to scanning beams
}

\author{
G. W. Forbes \\ The Institute of Optics, University of Rochester, Rochester, New York 14627
}

(Received 8 June 1993; accepted 2 December 1993)

From the Fresnel approximation to the Rayleigh-Sommerfeld solution for scalar wave propagation it follows that the field generated by a focused wave passing through an arbitrary aperture is simply related to the field generated by a collimated beam that is diffracted by any aperture of the same shape-the transverse intensity patterns are simply scaled and shifted. In fact, this result provides a simple, diffraction-based model for the action of a lens. A closed-form expression is found for the width of the beam generated by a focused uniform field that is diffracted by a circular aperture and, as an illustrative application, the beam of smallest maximum width over a fixed scanning depth is investigated.

\section{INTRODUCTION}

A conventional model in physical optics is based on the analysis of monochromatic scalar waves where the field can be written as $E(\mathbf{r}, t)=\operatorname{Re}\left\{U(\mathbf{r}) e^{-i \omega t}\right\}$. The amplitude and phases of the field are both contained in the complexvalued function $U(\mathbf{r})$ and, for $E(r, t)$ to be a solution of the (real) wave equation, $U(\mathbf{r})$ must satisfy the (complex) Helmholtz equation:

$$
\left\{\nabla^{2}+(\omega / c)^{2}\right\} U(\mathbf{r})=0
$$

The boundary values demanded of $U(\mathbf{r})$ complete the specification of a given problem and these distinguish classes of standard applications such as waveguide analysis or the modeling of laser cavities. The solution of interest here is the Rayleigh-Sommerfeld diffraction integral for free-space propagation: when the value of $U(\mathbf{r})$ is known on some fixed plane, say $z=0$, a two-dimensional convolution yields the field value on other planes. For many problems of interest, this solution is well approximated by ${ }^{1}$

$$
U(\mathbf{r}) \approx \frac{-i}{\lambda|\mathbf{r}|} \iint U\left(x^{\prime}, y^{\prime}, 0\right) e^{i k\left|\mathbf{r}-\left(x^{\prime}, y^{\prime}, 0\right)\right|} d x^{\prime} d y^{\prime},
$$

where the constants $k$ and $\lambda$ are defined by $k:=\omega / c$ and $\lambda:=2 \pi / k$. The Fresnel approximation puts this integral in a form that is the foundation for this work ${ }^{2}$

$$
\begin{gathered}
U(\mathbf{r}) \approx \\
\approx \frac{-i e^{i k|\mathbf{r}|}}{\lambda|\mathbf{r}|} \iint U\left(x^{\prime}, y^{\prime}, 0\right) \exp \left\{i k \left[\frac{1}{2}\left(x^{\prime 2}+y^{\prime 2}\right)\right.\right. \\
\left.\left.-x x^{\prime}-y y^{\prime}\right] /|\mathbf{r}|\right\} d x^{\prime} d y^{\prime} .
\end{gathered}
$$

The initial field value that is written in the integrands of Eqs. (1.2) and $(1.3)$ as $U\left(x^{\prime}, y^{\prime}, 0\right)$ is typically considered to result from a known incident field that is modified by the presence of a mask (say an aperture, a lens, or a transparency) characterized by a complex-valued transmission function $M(x, y)$. In this case, $U\left(x^{\prime}, y^{\prime}, 0\right)$ is written as $U\left(x^{\prime}, y^{\prime}, 0\right)=U_{\text {inc }}\left(x^{\prime}, y^{\prime}, 0\right) M\left(x^{\prime}, y^{\prime}\right)$. For the problems of interest here, $U\left(x^{\prime}, y^{\prime}, 0\right)$ is nonzero only in a region about the origin, and the extent of this region-say the nominal diameter-is written as $A$. Typical relative scales for optical problems are: $\lambda \approx 10^{-6} \mathrm{~m}, A \approx 10^{-4}-10^{-2} \mathrm{~m},|\mathbf{r}|$ is larger than a few times $A$, and for a given value of $|\mathbf{r}|$, $x^{2}+y^{2}$ is kept at least several times smaller than $|\mathbf{r}|^{2}$. With this, Eq. (1.3) gives an accurate approximation to the diffracted field and this setup is represented schematically in Fig. 1. The right-hand side of Eq. (1.3) is taken here to define the quantity that is sought so that, to avoid the appearance that new approximations are implied in what follows, this " $\approx$ " is henceforth replaced by " =".

The form of Eq. (1.3) suggests convenient coordinates for specifying $r$. Here, the angle coordinates of the spherical polars $(r, \theta, \phi)$ are replaced by the transverse Cartesian components $(x, y)$ and these coordinates are written collectively as $(x, y ; r)$. That is, a point is fixed here by specifying its distance from the origin-the nominal center of the diffracting mask-and the Cartesian components of the normal projection of the point onto the plane of the mask. As can be appreciated from Fig. 1, when the general struc- 